\title{
Avoiding the "set it and forget it mentality": A need to regularly reassess left ventricular assist device patients for optimal support
}

Jennifer A. Cowger, MD, MS, ${ }^{a}$ Gillian Grafton, DO, ${ }^{a}$ and Palak Shah, MD, MS ${ }^{\mathrm{b}}$

From ${ }^{a}$ Division of Cardiovascular Medicine, Department of Internal Medicine, Henry Ford Hospital, Detroit, Mich; and ${ }^{\mathrm{b}}$ Division of Cardiovascular Medicine, Department of Internal Medicine, Inova Heart \& Vascular Institute, Falls Church, Va.

Received for publication April 22, 2019; revisions received May 30, 2019; accepted for publication June 1, 2019; available ahead of print Oct 21, 2019.

Address for reprints: Jennifer A. Cowger, MD, MS, Henry Ford Hospitals, Detroit, MI 48202 (E-mail: jennifercowger@gmail.com).

J Thorac Cardiovasc Surg 2020;159:1322-5

$0022-5223 / \$ 36.00$

Copyright (C) 2019 Published by Elsevier Inc. on behalf of The American Association for Thoracic Surgery https://doi.org/10.1016/j.jtcvs.2019.06.134

Over the last 15 years, the field of mechanical circulatory support (MCS) has enjoyed a rapid technological evolution, leading to marked gains in patient survival. In 2010, average 1-year survival after left ventricular assist device (LVAD) implant was only $79 \%$ in patients enrolled into the Interagency Registry for Mechanically Assisted Circulatory Support. ${ }^{1}$ Presently, well-selected patients with end-stage systolic heart failure on continuous-flow, durable LVAD support attain average survivals of $83 \%$ to $88 \%$ at 1 year postoperatively, and approximately $50 \%$ of patients on permanent (destination therapy) LVAD support are alive at 5 years. $^{2,3}$ Despite these feats, however, LVAD therapy still carries great morbidity. Based on Interagency Registry for Mechanically Assisted Circulatory Support data, 80\% of patients experience at least 1 hospital readmission within 1 year of LVAD implant. ${ }^{2}$ Although readmission rates are highly variable, the average patient experiences 1.8 readmissions per year during the first year of support, improving marginally to $\sim 1.1$ readmissions per year thereafter. ${ }^{4}$ The most common causes of readmission during the first year include stroke $(9 \%-17 \%)$, mucocutaneous bleeding (20\%-25\%), infection (23\%-25\%), and/or persistent heart failure symptomatology $(12 \%-20 \%))^{2,5,6}$ Although costeffectiveness appears to be improving with each generation of device, systemic resource use is still high at $\$ 142,000$ to 171,000 USD per quality-adjusted life year. $^{7-9}$ Thus, to enable expansion of the field of MCS into the less ill (New York Heart Association Class IIIa, stage C) heart failure population, devices must become more costeffective with improved adverse event profiles and lower readmission rates.

Perhaps the simplest means of reducing readmission is to adequately treat the patient's heart failure through LVAD optimization. While patients undertake the heroics of LVAD support to improve heart failure symptoms and survival, $\sim 30 \%$ of patients after LVAD implant fail to attain

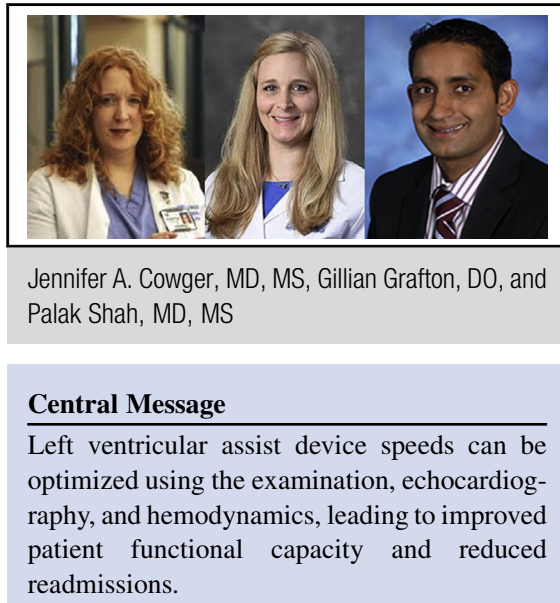

This Invited Expert Opinion provides a perspective on the following paper: Circ Heart Fail. 2019;12:e005094. https://doi.org/10 1161/CIRCHEARTFAILURE.118.005094.

See Commentary page on 1326.

a 6-minute walk test $>300 \mathrm{~m}$ or have a persistently poor quality of life 6 months after LVAD implant. ${ }^{10,11}$ In a study by Shah and colleagues ${ }^{12}$ of 71 patients on continuous-flow LVAD support, $29 \%$ of patients had persistently suboptimal hemodynamics a median 114 days after LVAD implantation, defined as a cardiac index (CI) $<2.2 \mathrm{~mL} / \mathrm{min} / \mathrm{m}^{2}$ and/or a pulmonary capillary wedge pressure (PCWP) $>18 \mathrm{~mm} \mathrm{Hg}$. Persistent or recurrent heart failure symptomatology after LVAD is often multifactorial and can be related to new or worsening right ventricular (RV) failure, aortic insufficiency, arrhythmias, concomitant comorbidities, or insufficient LVAD support of left-sided heart failure. $^{10,12-14}$ Thus, it is imperative that practitioners regularly reassess and optimize LVAD settings, patient blood pressure, and volume status to ensure that they are getting the most of what their device has to offer.

\section{RECOMMENDED STRATEGIES TO ENSURE PATIENTS ARE OPTIMIZED ON LVAD SUPPORT}

At the time of LVAD implant, LVAD settings in the operative suite should be guided by intraoperative cardiopulmonary hemodynamics and findings on transesophageal echocardiogram, both of which are heavily influenced by 
the preoperative stability of the patient and perioperative RV dysfunction. While normalizing cardiac output via LVAD support can improve end-organ perfusion, overly rapid or aggressive LVAD support early postoperatively can lead to adverse septal shift, increased RV wall stress, and subsequent RV failure. Thus, in the early postoperative period while patients are still on inotropes, it is often advantageous to set LVAD flows slightly below "ideal" to allow the RV to adapt to LVAD support, later increasing LVAD speeds as volume status nears euvolemia and inotropes are discontinued. Thereafter, for most stable patients, we feel a good rule of thumb is to set device flow based on body surface area, aiming to achieve an indexed LVAD flow of $2.2 \mathrm{~mL} / \mathrm{min} / \mathrm{m}^{2}$. While a $5^{\prime} 8^{\prime \prime}$ patient weighing 180 pounds (body surface area 1.9), for example, may do well with $4.3 \mathrm{~L}$ of resting LVAD flow, a 6'2" patient weighing 240 pounds (body surface area 2.35 ) will likely require $>5 \mathrm{~L}$ of LVAD flow to achieve optimal functional capacity and euvolemia as an outpatient. Echocardiography-guided LVAD speed adjustment should be used to assist with achieving optimal left ventricular (LV) dimensions, septal positioning, RV function, and aortic valve-opening frequency. ${ }^{13}$ Before discharge, when patients are near euvolemia and off inotropic support, we recommend obtaining a repeat echocardiogram to reassess the response of the right and left ventricles to the LVAD settings intended for outpatient support.

\section{ENSURING LVAD OUTPATIENTS ARE OPTIMIZED}

Outside of the intensive care unit, optimizing patients on LVAD support is complicated by lack of a fast and accurate means of assessing patient cardiopulmonary hemodynamics. LVAD technologies by design lead to preferential reduction in PCWP with minimal impact on right atrial pressure. ${ }^{15}$ Thus, the jugular venous pulse assessment during a clinic encounter may be a poor surrogate for LV filling pressures. While device pulsatility index (HeartMate II and HeartMate 3; Abbott, Abbott Park, Ill), flow waveforms (HVAD; Medtronic, Minneapolis, Minn), and echocardiography-guided LVAD optimization are integral to LVAD patient management, we do not feel that they can divulge filling pressures with reasonable accuracy and precision. Some investigators have shown correlation between diastolic filling patterns (E/A and $\mathrm{E} / \mathrm{E}^{\prime}$ ) on echocardiography ${ }^{16}$ and/or the slope of the HVAD waveform and LV filling pressures ${ }^{17}$ but the interrater reliability of these tools in nonexpert hands has not been convincingly demonstrated to allow for routine use. When echocardiography is performed to optimize LVAD speed, we feel it is also critical to appreciate the differences in the response of the LV to speed increases between LVAD models. Using very elegant 3-dimensional echocardiography, Addetia and colleagues ${ }^{18}$ and Sauer and colleagues ${ }^{19}$ have shown that LV geometry differs in response to axial flow and centrifugal flow echocardiography-guided device speed changes, with the axial flow HeartMate II device causing more prominent decompression of the LV (LV becomes less spherical and more conical) as measured by a reduction in the LV internal diastolic diameter (LViDd). ${ }^{18,19}$ In contrast, the LV in patients supported on the intrapericardial, hydrodynamic centrifugal flow HVAD remains spherical during device ramping, with less prominent LViDd reductions. ${ }^{18,19}$ Further, we caution extrapolating LViDd to LV filling pressure. In a study of patients with a HeartMate II device, LViDd was found to correlate poorly with PCWP on invasive hemodynamic assessment. ${ }^{20}$ As such, an LViDd of $70 \mathrm{~mm}$ in a patient on HeartMate II support does not necessarily imply that the patient has persistently high left-sided filling pressures. Rather, filling pressures may still be low despite the absence of ventricular remodeling.

Given the aforementioned limitations of the examination and echocardiography, we feel the most reliable means of optimizing patient filling pressures and device support is by right heart catheterization. Right heart catheterization is a quick, low-risk procedure that can be completed in patients with LVAD using a 5- to 7-F venous sheath and balloon-tipped catheter without the need to interrupt anticoagulation and often without need for fluoroscopy. Echocardiography assessment simultaneous with the heart catheterization procedure is complementary and should strongly be considered in patients with very aberrant hemodynamics or for whom large changes in device speed are being made. Shah and colleagues ${ }^{12}$ showed that Swan-Ganzguided LVAD speed adjustment to achieve optimal CI $\left(>2.2 \mathrm{~mL} / \mathrm{min} / \mathrm{m}^{2}\right)$ and PCWP $(\leq 18 \mathrm{~mm} \mathrm{Hg})$ was feasible in $68 \%$ of patients and resulted in significant gains in patient 6-minute walk test distance (from $338 \mathrm{~m}$ preoptimization to $354 \mathrm{~m}$ after optimization). A prospective multicenter pilot study randomizing patients with LVAD to postoperative Swan-Ganz hemodynamic-guided device optimization versus standard care showed that hemodynamic-guided device optimization (defined as right atrial pressure $<12 \mathrm{~mm} \mathrm{Hg}$, PCWP $<18 \mathrm{~mm} \mathrm{Hg}$, and CI $>2.2 \mathrm{~L} / \mathrm{min} / \mathrm{m}^{2}$ ) led to nonsignificant reductions in heart failure symptoms and hemocompatibility-related adverse events. $^{21}$ Similar to the study by Shah and colleagues, $67 \%$ of patients required changes in LVAD speed settings to achieve optimal hemodynamics. In another study of 88 patients on LVAD support, hemodynamic-guided LVAD speed changes were associated with a reduction in overall hospital readmission (1.15 vs 2.86 events/year), driven largely by fewer heart failure hospitalizations $(0.08$ vs 0.71 events/year, $P=.016) .{ }^{14}$

Although we do not feel that all patients on LVAD support require routine right heart catheterization on a protocolized basis, we feel the aforementioned studies support adopting a much lower threshold for elective hemodynamic (re)assessment after LVAD implant. In patients with 
significant RV failure during the index LVAD implant, we feel it is wise to reassess cardiopulmonary hemodynamics 3 to 6 months after surgery to allow for further optimization of device speeds, vasodilators, and/or diuretic therapies. If significant changes in therapies are made, a follow-up echocardiogram or hemodynamic assessment in 1 to 3 months may be necessarily. We also feel that right heart catheterization should be strongly considered in patients with persistent HF symptomatology (New York Heart Association III-IV symptoms, $<300 \mathrm{~m}$ achieved on a 6-minute walk test distance due to dyspnea) and/or high diuretic needs at 3 to 6 months or more postoperative, regardless of echo findings. Finally, the presence of inappropriately low device flows for set speed, recurrent low flow alarms without clear cause, or persistent/recurrent unexplained dizziness at any point after LVAD implant should foster a low threshold for hemodynamic assessment, looking for signs of device dysfunction (including outflow obstruction). While autonomic dysfunction during LVAD support is a known phenomenon leading to patient orthostasis, we have also found that some patients on HeartMate 3 support (especially elderly patients and patients with long-standing diabetes) with normal device function are particularly sensitive to the combined state of dehydration and excessive afterload reduction. The "dizziness" symptomatology in dehydrated patients on HeartMate 3 support is likely exacerbated by the device's pulsatility algorithm, with transient cerebral hypoperfusion occurring during programmed speed reductions that occur coincident with ventricular systole. Since many patients on HeartMate 3 support display wider systemic arterial pulse pressures than those measured in patients on other continuous-flow devices, overtreatment of blood pressure may result when Doppler opening pressure is assumed to approximate mean arterial pressure. While dizziness often responds to reductions in diuretics and vasodilators, hemodynamic assessment with a fluid challenge when clinically appropriate may benefit patients with persistent symptoms or identify other sources of symptomatology.

In conclusion, the field of MCS should be commended for the rapid and large gains achieved in patient survival and device durability. However, the high rates of readmission, persistent/recurrent heart failure, and the aforementioned combined results suggest that advanced heart failure practitioners are not doing enough to ensure patients remain optimized during chronic LVAD support. Marrying results from echocardiography, device interrogations, and hemodynamic monitors (such as CardioMEMS; Abbott Inc) may be one means of noninvasively optimizing patients with LVAD. Alternatively, these studies suggest practitioners should not hesitate to undertake right heart catheterization in patients who have persistent heart failure symptomatology, low device flows, or high diuretic requirements. It is time to move from a "set it and forget it" to a "set it and reassess it" management plan for patients on chronic LVAD support.

\section{Conflict of Interest Statement}

Dr Cowger is a paid speaker for Abbott and Medtronic. She is a member of scientific steering committees for Medtronic and Procyrion. Henry Ford receives research-related support from Medtronic and Abbott. Dr Shah reports grant support from American Heart Association/Enduring Hearts Scientist Development Grant, Merck, Haemonetics for unrelated research, and consulting for NuPulse $\mathrm{CV}$ and Ortho Clinical Diagnostics. Dr Grafton has nothing to disclose with regard to commercial support.

\section{References}

1. Kirklin JK, Naftel DC, Kormos RL, Stevenson LW, Pagani FD, Miller MA, et al Third INTERMACS annual report: the evolution of destination therapy in the United States. J Heart Lung Transplant. 2011;30:115-23.

2. Kormos RL, Cowger J, Pagani FD, Teuteberg JJ, Goldstein DJ, Jacobs JP, et al. The Society of Thoracic Surgeons INTERMACS database annual report: evolving indications, outcomes, and scientific partnerships. Ann Thorac Surg. 2019;107:341-53.

3. Mehra MR, Goldstein DJ, Uriel N, Cleveland JC Jr, Yuzefpolskaya M, Salerno C, et al. Two-year outcomes with a magnetically levitated cardiac pump in heart failure. N Engl J Med. 2018;378:1386-95.

4. Vidula H, Kutyifa V, Johnson BA, Strawderman RL, Harrington D, Polonsky B, et al. Readmission patterns during long-term follow-up after left ventricular assist device implantation. Am J Cardiol. 2018;122:1021-7.

5. Colombo PC, Mehra MR, Goldstein DJ, Estep JD, Salerno C, Jorde UP, et al. Comprehensive analysis of stroke in the long-term cohort of the MOMENTUM 3 study. Circulation. 2019;139:155-68.

6. Milano CA, Rogers JG, Tatooles AJ, Bhat G, Slaughter MS, Birks EJ, et al HVAD: the ENDURANCE supplemental trial. JACC Heart Fail. 2018;6: 792-802.

7. Rogers JG, Bostic RR, Tong KB, Adamson R, Russo M, Slaughter MS. Costeffectiveness analysis of continuous-flow left ventricular assist devices as destination therapy. Circ Heart Fail. 2012;5:10-6.

8. Tadmouri A, Blomkvist J, Landais C, Seymour J, Azmoun A. Cost-effectiveness of left ventricular assist devices for patients with end-stage heart failure: analysis of the French hospital discharge database. ESC Heart Fail. 2018;5:75-86.

9. Baras Shreibati J, Goldhaber-Fiebert JD, Banerjee D, Owens DK, Hlatky MA. Cost-effectiveness of left ventricular assist devices in ambulatory patients with advanced heart failure. JACC Heart Fail. 2017;5:110-9.

10. Cowger JA, Naka Y, Aaronson KD, Horstmanshof D, Gulati S, RindeHoffman D, et al. Quality of life and functional capacity outcomes in the MOMENTUM 3 trial at 6 months: a call for new metrics for left ventricular assist device patients. J Heart Lung Transplant. 2018;37:15-24.

11. Arnold SV, Jones PG, Allen LA, Cohen DJ, Fendler TJ, Holtz JE, et al. Frequency of poor outcome (death or poor quality of life) after left ventricular assist device for destination therapy: results from the INTERMACS registry. Circ Heart Fail. 2016;9(8).

12. Shah P, Badoe N, Phillips S, Abdullah K, May CW, Nabut JL, et al. Unrecognized left heart failure in LVAD recipients: the role of routine invasive hemodynamic testing. ASAIO J. 2018;64:183-90.

13. Uriel N, Morrison KA, Garan AR, Kato TS, Yuzefpolskaya M, Latif F, et al. Development of a novel echocardiography ramp test for speed optimization and diagnosis of device thrombosis in continuous-flow left ventricular assist devices: the Columbia ramp study. J Am Coll Cardiol. 2012;60:1764-75.

14. Imamura T, Jeevanandam V, Kim G, Raikhelkar J, Sarswat N, Kalantari S, et al. Optimal hemodynamics during left ventricular assist device support are associated with reduced readmission rates. Circ Heart Fail. 2019;12:e005094.

15. Uriel N, Sayer G, Addetia K, Fedson S, Kim GH, Rodgers D, et al. Hemodynamic ramp tests in patients with left ventricular assist devices. JACC Heart Fail. 2016; 4:208-17.

16. Estep JD, Vivo RP, Krim SR, Cordero-Reyes AM, Elias B, Loebe M, et al. Echocardiographic evaluation of hemodynamics in patients with systolic heart failure supported by a continuous-flow LVAD. J Am Coll Cardiol. 2014;64:1231-41. 
17. Lai JV, Muthiah K, Macdonald PS, Jansz P, Hayward CS. Estimation of left ventricular assist device pre-load using pump flow waveform analysis. J Heart Lung Transplant. 2017;36:240-2.

18. Addetia K, Uriel N, Maffessanti F, Sayer G, Adatya S, Kim GH, et al. 3D morphological changes in LV and RV during LVAD ramp studies. JACC Cardiovasc Imaging. 2018;11(2 Pt 1):159-69.

19. Sauer AJ, Meehan K, Gordon R, Abicht T, Rich JD, Anderson AS, et al. Echocardiographic markers of left ventricular unloading using a centrifugal-flow rotary pump. J Heart Lung Transplant. 2014;33 449-50.

20. Jung MH, Hassager C, Balling L, Russell SD, Boesgaard S, Gustafsson F. Relation between pressure and volume unloading during ramp testing in patients supported with a continuous-flow left ventricular assist device. ASAIO J. 2015;61:307-12.

21. Uriel N, Burkhoff D, Rich JD, Drakos SG, Teuteberg JJ, Imamura T, et al. Impact of hemodynamic ramp test-guided HVAD speed and medication adjustments on clinical outcomes. Circ Heart Fail. 2019;12:e006067. 